\title{
Análisis del Conocimiento de la Terminología Anatómica Internacional (TAI) por el Cirujano General
}

\author{
Analysis of the General Surgeon's Knowledge of International Anatomical Terminology (IAT)
}

Rubén D. Algieri*; María Soledad Ferrante* \& Eduardo Pró*

ALGIERI, R. D.; FERRANTE, M. S. \& PRÓ, EAnálisis del Conocimiento de la Terminología Anatómica Internacional (TAI) Por el Cirujano General. Int. J. Morphol., 31(4):1511-1516, 2013.

RESUMEN: Hace más de un siglo que se llevan adelante constantes discusiones internacionales para lograr unificar criterios respecto al lenguaje universal en Anatomía, buscando priorizar básicamente un término sobre otros equivalentes, mediante la elección de una nominación única para designar un solo concepto. En el último esfuerzo por consensuar los términos anatómicos, se creó la "Terminología Anatómica Internacional" (TAI) que actualmente ha desplazado a la anteriormente llamada "Nómina Anatómica". Estudio estadístico retrospectivo a través de una encuesta anónima a 96 médicos especialistas en Cirugía General que se realizó en la III Cátedra de Anatomía de la Facultad de Medicina de la Universidad de Buenos Aires, durante el período de mayo/2005 a mayo/2006. Se consideraron los años de graduado y de especialista, el conocimiento de las nomenclaturas anatómicas y la actualización bibliográfica en la especialidad. Luego fue comparada con la repetición de dicha encuesta a 92 médicos especialista en Cirugía General, durante el período julio 2010 a julio 2011. De los 96 médicos especialistas en Cirugía General encuestados en el primer período, 30 (31,25\%) desconocía la existencia de un listado oficial de nombres de estructuras anatómicas y 66 (68,75\%) conocía su existencia. De estos 66 que conocían la existencia de un listado oficial, 60(90,91\%) presentó dificultades para la denominación de dicho listado y $6(9,09 \%)$ enunciaron correctamente a la TAI. De los 92 médicos especialistas en Cirugía General encuestados en el segundo período, 9 (9,78\%) desconocía la existencia de un listado oficial de nombres de estructuras anatómicas y $83(90,22 \%)$ conocía su existencia. Al interrogarlos a los 83 sobre la denominación oficial del listado de nombres de estructuras anatómicas, 32 (38,55\%) presentó dificultades para la denominación de dicho listado y $51(61,45 \%)$ enunciaron correctamente a la TAI. En lo que respecta a Cirugía General, hemos visto que con el pasar de los años aumentó el número de médicos especialistas que tomaron conocimiento de la existencia de un listado oficial de nombres de estructuras anatómicas, y también los que comenzaron a implementar la Terminología Anatómica Internacional como fuente oficial de términos anatómicos, pero todavía sigue siendo mayoría los que desconocen su existencia o su nombre. Por lo que es necesario trabajar en este aspecto, con el fin de incentivar la actualización permanente y lograr así, unificar los términos, facilitando la enseñanza y aprendizaje, y evitando confusiones en la comunicación científica no sólo entre los diferentes lugares del mundo sino entre profesionales médicos especialistas de distintos grupos etarios y/o de diferentes años de graduado. Siendo los especializados en disciplinas morfológicas y quienes los aplican en la actividad cotidiana, los responsables de estimular su conocimiento.

PALABRAS CLAVE: Terminologia Anatomica Internacional; Médico cirujano; Cirugía general; Nivel de conocimiento; Términos anatómicos.

\section{INTRODUCCIÓN}

Es frecuente en todo tipo de lenguaje la existencia de varios términos para designar un mismo concepto (sinonimia) y además, la posibilidad de que un mismo término pueda tener varios significados (polisemia) (Díaz, 2001; Jiménez, 2009; Salgado \& Trujillo, 2010). Esto es común en el idioma científico y médico, con mayor razón en las ciencias morfológicas y de ellas, la anatomía es particularmente la que en términos prácticos genera gran número de controversias (Acuña et al., 2007) y desencuentros. A la gran cantidad de términos anatómicos existentes, se suman las con- fusiones originadas cuando en algunos países y en otras ocasiones, traductores de escritos anatómicos, le asignan un nombre diferente a cada estructura o elemento. Así, nos encontraríamos frente a "un término" expresado de diferentes formas. Ya hace más de un siglo que se llevan adelante constantes discusiones internacionales para lograr unificar criterios respecto al lenguaje universal en Anatomía, buscando priorizar básicamente un término sobre otros equivalentes, mediante la elección de una nominación única para designar un solo concepto (Díaz; Salgado \& Trujillo; W-ster, 
1998). En el último esfuerzo por consensuar los términos anatómicos, se creó la "Terminología Anatómica Internacional" que actualmente ha desplazado a la anteriormente llamada "Nómina Anatómica". Siendo el objetivo, analizar el empleo de la Terminología Anatómica Internacional (TAI) a través de un estudio comparativo en el tiempo, a fin de observar la evolución del conocimiento de la misma como fuente oficial de términos anatómicos por el médico especialista en cirugía general.

\section{MATERIAL Y MÉTODO}

Se realizó en la III Cátedra de Anatomía de la Facultad de Medicina de la Universidad de Buenos Aires, durante el período de mayo/2005 a mayo/2006, un estudio estadístico retrospectivo a través de una encuesta anónima a 96 médicos especialistas en Cirugía General. Se consideraron los años de graduado y de especialista, el conocimiento de las nomenclaturas anatómicas y la actualización bibliográfica en la especialidad. Situación que fue comparada con la repetición de dicha encuesta a 92 médicos especialista en Cirugía General, durante el período julio 2010 a julio 2011.

\section{RESULTADOS}

De los 96 médicos especialistas en Cirugía General encuestados en el primer período, 30 $(31,25 \%)$ desconocía la existencia de un listado oficial de nombres de estructuras anatómicas y 66 $(68,75 \%)$ conocía su existencia (Fig. 1A). Al interrogarlos a los 66 sobre la denominación oficial del listado de nombres de estructuras anatómicas, $60(90,91 \%)$ presentó dificultades para la denominación de dicho listado (siendo $31(51,67 \%)$ los que nombraron a la Nómina Anatómica, 18 (30\%) no recordaban el nombre del listado, 11 (18,33\%) la denominaron Nómina Francesa) y $6(9,09 \%)$ enunciaron correctamente a la Terminología Anatómica Internacional (Figs. 2A y 3A). Del total de los encuestados (96), 6 (6,25\%) identificaron correctamente las estructuras anatómicas de la región, según la Terminología Anatómica Internacional, sobre gráficos de la región inguinal. Respecto a la relación con el tiempo de graduado, se evidenció en los graduados con 6-10 años, $29(30,21 \%)$ que 6 $(20,69 \%)$ enunciaron correctamente a la TAI y 17
A

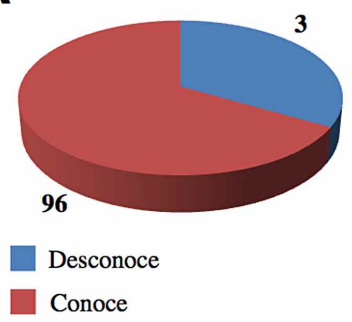

B

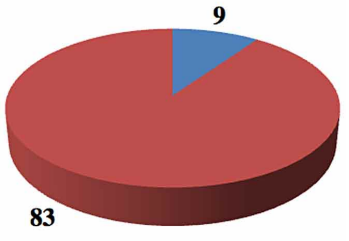

$9,78 \%$ desconoce

$90,22 \%$ conoce

Fig. 1. Resultados de encuestas sobre el conocimiento de la existencia de un listado oficial de nombres de estructuras anatómicas. A. Primer período: 96 encuestados. B. Segundo período: 92 encuestados.

A

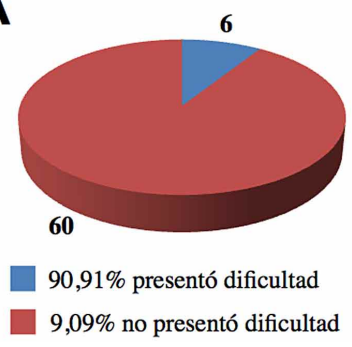

B

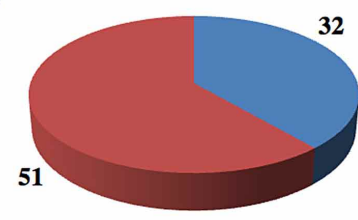

$38,55 \%$ presentó dificultad

$61,45 \%$ no presentó dificultad

Fig. 2. Resultados de encuestas del primer período sobre la existencia de dificultades en la denominación del listado oficial de nombres de estructuras anatómicas. A. Resultados sobre los 66 encuestados que conocía su existencia en el 1er período. B. Resultados sobre los 83 encuestados que conocía su existencia en el $2^{\circ}$ período.

$(58,62 \%)$ conocían la existencia del listado oficial de nombres de estructuras anatómicas (de los cuales 11(64.71\%) la nombraron Nómina anatómica, $4(23,53 \%)$ no recordaba su nombre y 2 $(11,76 \%)$ la denominaron Nómina Francesa) y $6(20,69 \%)$ la desconocían (Fig. 4 I.A). En el grupo de 11-15 años, 31 (32,29\%), 22 $(70,97 \%)$ conocían la existencia del listado oficial de nombres de estructuras anatómicas (de los cuales 12 (54,55\%) la nombraron Nómina Anatómica, $6(27,27 \%)$ no recordaba su nombre y 4 $(18,18 \%)$ la denominaron Nómina Francesa) y $9(29,03 \%)$ la desconocían (Fig. 4 I.B). En el grupo de más de 16 años, 36 (37,50\%), $21(58,33 \%)$ de los encuestados conocían la existencia del listado oficial de nombres de estructuras anatómicas (de los cuales, 8 $(38,10 \%)$ la nombraron Nómina Anatómica, $8(38,10 \%)$ no recordaba su nombre y $5(23,80 \%)$ la denominaron Nómina Francesa) y $15(41,67 \%$ ) la desconocían (Fig. 4 I.C). La última actualización sobre anatomía de la región referida correspondió, dentro de los 1-5 años en 16 de los encuestados (16,67\%), 6-10 años en $27(28,12 \%), 11-15$ años en $28(29,17 \%)$ y mas de 16 años en $25(26,04 \%)$ (Fig. 5).

De los 92 médicos especialistas en Cirugía General encuestados en el segundo período, 9 (9,78\%) desconocía la existencia de un listado oficial de nombres de estructuras anatómicas 
A

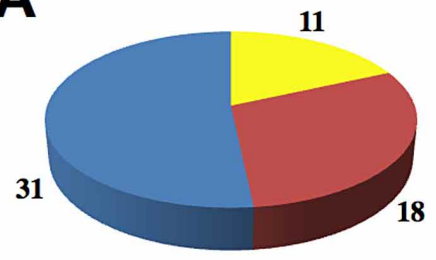

$\mathbf{5 1 , 6 7 \% ~ d e n o m i n o ́ ~ c o m o ~ N o ́ m i n a ~ A n a t o ́ m i c a ~}$

$12,5 \%$ no recordaban nombre de listado oficial

18,33\% denominó como Nómina Francesa
B

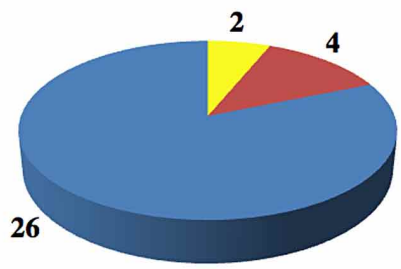

81,25\% denominó como Nómina Anatómica

$12,5 \%$ no recordaban nombre de listado oficial

6,25\% denominó como Nómina Francesa

Fig. 3. A. Resultados de encuestas a los 60 médicos especialistas en cirugía que presentaron dificultades al denominar el listado oficial de nombres de estructuras anatómicas en el $1^{\circ}$ período. B. Resultados de encuestas a los 32 médicos especialistas en cirugía que presentaron dificultades al denominar el listado oficial de nombres de estructuras anatómicas en el $2^{\circ}$ período.

I. A

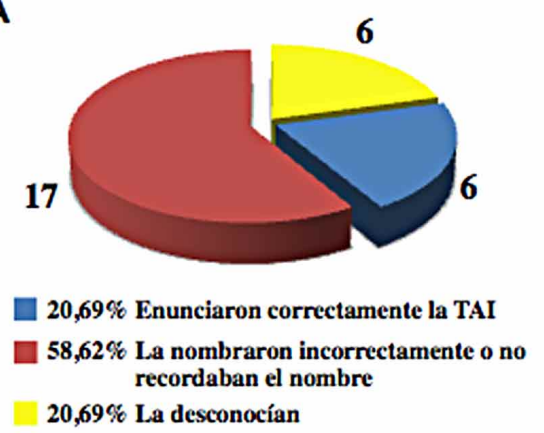

I. B

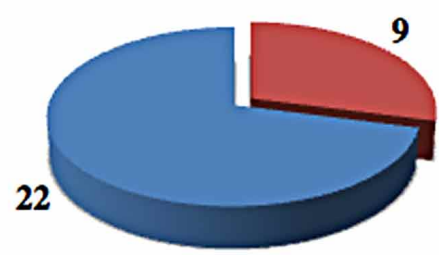

70,97\% Conocían la existencia de listado pero no recordaban su nombre o era incorrecto

$29,03 \%$ La desconocían

I. C

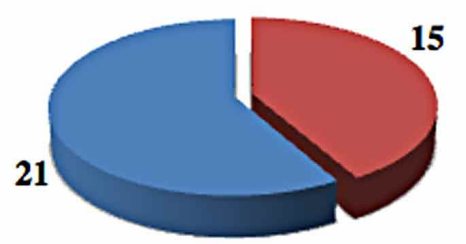

$\mathbf{5 8 , 3 3 \%}$ Conocían la existencia de listado pero no recordaban su nombre o era incorrecto
II. A

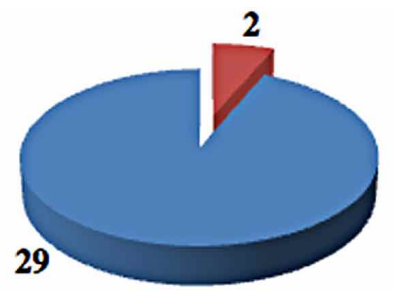

93,55\% Enunciaron correctamente la TAI

$58,62 \%$ La nombraron incorrectamente

II. B

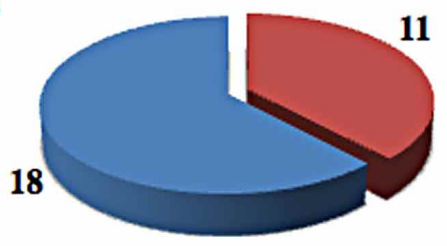

$62,07 \%$ Enunciaron correctamente la TAI

$37,93 \%$ La nombraron incorrectamente

II. C

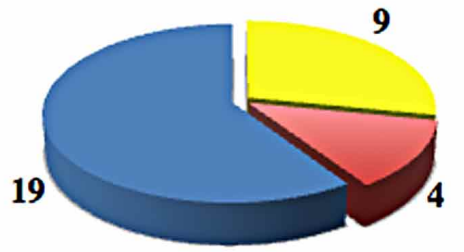

$\mathbf{5 9 , 3 7 \% ~ C o n o c i ́ a n ~ l a ~ e x i s t e n c i a ~ d e ~ l i s t a d o ~ p e r o ~}$ no recordaban su nombre o era incorrecto

12,5\% Enunciaron correctamente la TAI

28,31\% La desconocían

Fig. 4. Resultados de encuestas respecto al listado oficial de nombres de estructuras anatómicas (TAI) en relación con el tiempo de graduado. I.A. 6-10 años de graduado (1er período, 29 médicos especialistas en cirugía); I.B. 11-15 años de graduado (1er período, 31 médicos especialistas en cirugía); I.C. >16 años de graduado (1er período, 36 médicos especialistas en cirugía); II. A. 6-10 años de graduado (2 período, 31 médicos especialistas en cirugía); II.B. 11-15 años de graduado ( $2^{\circ}$ período, 29 médicos especialistas en cirugía); II.C. > 16 años de graduado ( $2^{\circ}$ período, 32 médicos especialistas en cirugía). 
y $83(90,22 \%)$ conocía su existencia (Fig. 1B). Al interrogarlos a los 83 sobre la denominación oficial del listado de nombres de estructuras anatómicas, 32 (38,55\%) presentó dificultades para la denominación de dicho listado (siendo $26(81,25 \%)$ los que nombraron a la Nómina Anatómica, 4 $(12,50 \%)$ no recordaban el nombre, $2(6,25 \%)$ la denominaron Nómina Francesa y $51(61,45 \%)$ enunciaron correctamente a la Terminología Anatómica Internacional (Figs. 2B y 3B). Del total de los encuestados (92), $51(55,43 \%)$ identificaron correctamente las estructuras anatómicas de la región, según la Terminología Anatómica Internacional, sobre gráficos de la región inguinal. Respecto a la relación con el tiempo de graduado, se evidenció en los graduados con 6-10 años, $31(33,70 \%)$ que $29(93,55 \%)$ de los encuestados enunciaron correctamente a la TAI y $2(6,45 \%)$ la nombraron Nómina anatómica (Fig. 4 II.A.). En el grupo de 11-15 años, 29 (31,52\%), 18 (62,07\%) enunciaron correctamente a la TAI, y $11(37,93 \%)$ conocían la existencia del listado oficial de nombres de estructuras anatómicas (de los cuales $10(90,91 \%)$ la nombraron Nómina Anatómica y $1(9,09 \%)$ no recordaba su nombre) (Fig. 4 II.B). En el grupo de más de 16 años, $32(34,78 \%)$ encuestados, 4 (12,5\%) enunciaron correctamente la TAI, $19(59,37 \%)$ conocían la existencia del listado oficial de nombres de estructuras anatómicas (de los cuales, 14 (73,68\%) la nombraron Nómina Anatómica, 3(15,79\%) no recordaba su nombre y $2(10,53 \%)$ la denominaron Nómina Francesa) y $9(28,13 \%)$ la desconocían (Fig. 4 II.C.). La última actualización sobre anatomía de la región referida correspondió, dentro de los 1-5 años en 51 de los encuestados (55,43\%), 6-10 años en 20 $(21,74 \%), 11-15$ años en $11(11,96 \%)$ y mas de 16 años en 10 (10,87\%) (Fig. 5).

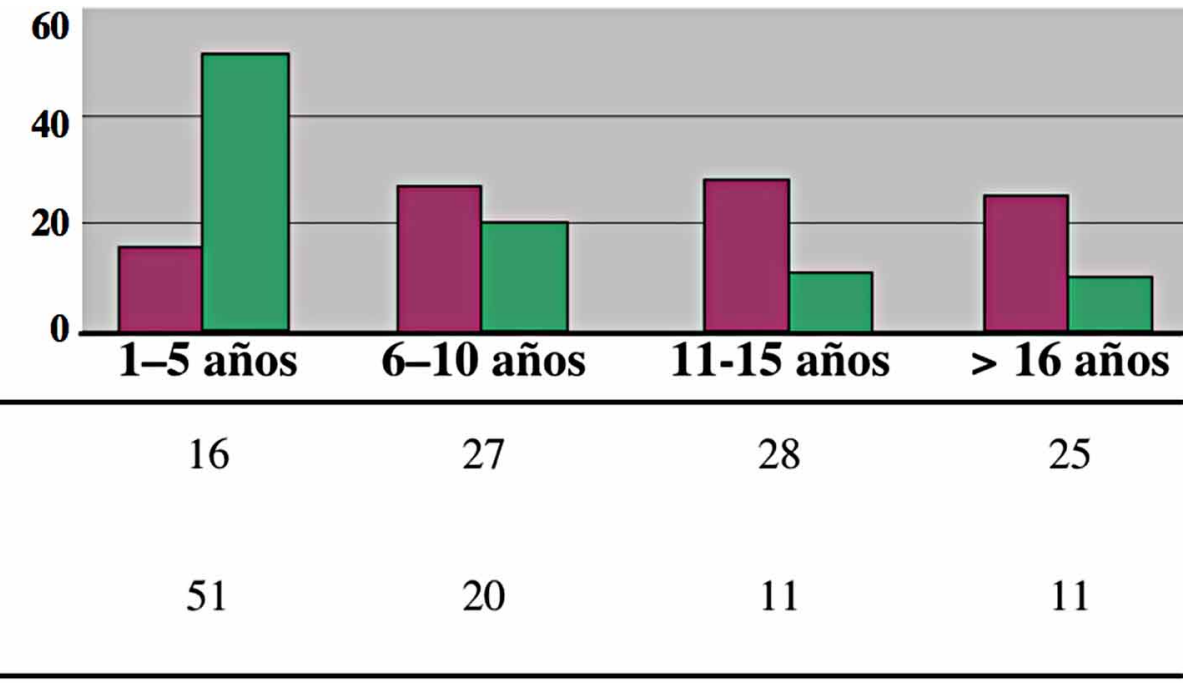

Fig. 5. Resultados de encuestas respecto a años de última actualización en Anatomía de la región encuestada.

\section{DISCUSIÓN}

Los términos anatómicos surgen de la prehistoria (Acuña et al.; Cruz Gutiérrez et al., 2010). Desde hace muchos años se viene trabajando para lograr unificar los criterios terminológicos, con el fin de obtener una comunicación clara y precisa en ciencias de la salud (Cruz Gutiérrez et al.). En 1895, Wilhem Hiss creó la comisión de Anatomistas en el Congreso de la Sociedad Alemana Anatómica, al cual se le encomendó crear un listado de términos anatómicos como código anatómico en Basilea, Suiza, que se denominó Nómina Anatómica de Basilea (BNA). Se caracterizó por ser simple y breve en sus nombres que debían ser únicos por estructura, los cuales estaban en latín como lengua oficial, y se suspendieron los epónimos (Acuña et al.; Algieri et al., 2010a; Algieri et al., 2010b; Algieri et al., 2011a; Álvarez \& Campohermoso, 2007; Salgado \& Trujillo).

En 1903 se funda la International Federation of Associations of Anatomists (IFAA: Federación Internacional de Asociaciones de Anatomistas) reuniéndose por primera vez en ese año en Ginebra, Suiza (Acuña et al.; Algieri et al., 2010a). En el $6^{\circ}$ Congreso Internacional de Anatomía, en París, en el año 1955, se realizó su corrección y 
agregados; se adoptó una nomenclatura latina basada en la BNA denominada: París Nómina Anatómica (PNA), y se formó el Comité de Nomenclatura Anatómica Internacional (IANC) (Acuña et al.; Algieri et al., 2010a; Algieri et al., 2011a; Álvarez \& Campohermoso; Cruz Gutiérrez et al.; International Anatomical Nomenclature Committee, 1961, 1968). Se fueron realizando diferentes modificaciones hasta que en 1989 el Comité Internacional de Nomenclatura Anatómica (IANC) publicó la sexta edición de la Nómica Anatómica (sin revisión por la IFAA), mientras tanto se establecía el Comité Federativo de Terminología Anatómica Internacional (FICAT), en Río de Janeiro (Acuña et al.; International Anatomical Nomenclature Committee, 1989).

Los integrantes del FICAT publicaron en 1998 el nuevo listado al que denominaron Terminología Anatómica Internacional (TAI), con términos en latín y sus equivalentes en inglés, reemplazando a las anteriores. Consta de 7583 términos anatómicos, de los cuales 1000 de ellos son nuevos. Basado en ésta Terminología Internacional en latín, cada idioma puede formular su propia nomenclatura. Si bien se desaconseja el uso de epónimos, existe un apéndice con los más frecuentes (Acuña et al.; Algieri et al., 2010a, 2010b, 2011a, 2011b; Álvarez \& Campohermoso; Federative Committee on Anatomical Terminology, 1998; ManzanaresCéspedes, 2010; Martin et al., 2009, 2010; Matusz, 2010; Vogl, 2009; Whitemore, 2009). A partir del 2001 la Sociedad Anatómica Española tradujo esta Terminología Anatómica Internacional al idioma español y se encuentra publicado por Ed. Médica Panamericana/Madrid (Algieri et al., 2010a, 2011a, 2011b). En 2006, se reunieron ocho expertos en Terminología Anatómica en la 29 a Reunión del FICAT, perteneciente al IFAA, quienes continúan trabajando permanentemente en la actualización de la misma (Pawlina \& Drake, 2009).

Durante diferente períodos se fueron realizando en el Departamento de Anatomía de la Facultad de Medicina de la Universidad de Buenos Aires, varios estudios estadísticos retrospectivo a través de una encuestas anónimas a médicos especialistas en Cirugía General; donde se consideraron los años de graduado y de especialista, el conocimiento de las nomenclaturas anatómicas y la actualización bibliográfica sobre distintas regiones anatómicas, donde se fueron evaluando los porcentajes de médicos especialistas que desconocían la existencia de un listado oficial de nombres de estructuras anatómicas, aquellos que conocían su existencia y los que identificaron correctamente las estructuras de la región considerada, según la Terminología Anatómica Internacional. En algunos de ellos se vio que la actualización bibliográfica sobre la región en los últimos 5 años, condujo al conocimiento de la Terminología Anatómica Internacional (Algieri et al., 2010a, 2011b, 2011b).

\section{CONCLUSION}

En lo que respecta a Cirugía General, hemos visto que con el pasar de los años aumentó el número de médicos especialistas que tomaron conocimiento de la existencia de un listado oficial de nombres de estructuras anatómicas, y también los que comenzaron a implementar la Terminología Anatómica Internacional como fuente oficial de términos anatómicos, pero todavía sigue siendo mayoría los que desconocen su existencia o su nombre. Por lo que es necesario trabajar en este aspecto, con el fin de incentivar la actualización permanente y lograr así, unificar los términos, facilitando la enseñanza y aprendizaje, y evitando confusiones en la comunicación científica no sólo entre los diferentes lugares del mundo sino entre profesionales médicos especialistas de distintos grupos etarios y/o de diferentes años de graduado. Siendo los especializados en disciplinas morfológicas y quienes los aplican en la actividad cotidiana, los responsables de estimular su conocimiento.

ALGIERI, R. D.; FERRANTE, M. S. \& PRÓ, E. Analysis of the General Surgeon's knowledge of International Anatomical Terminology (IAT). Int. J. Morphol., 31(4):1511-1516, 2013.

SUMMARY: Ongoing international discussions have been carried out for over a century in order to coordinate criteria regarding universal Anatomy language prioritizing the search for an equivalent term by choosing only one nomination to designate a single concept. In the latest effort to agree on anatomical terms the "International Anatomical Terminology" (IAT) which replaced the previously called "Anatomical Nomination". A retrospective statistical study was carried out through an anonymous survey of 96 medical specialists in General Surgery at the anatomy chairmanship of the Faculty of Medicine, University of Buenos Aires during May/2005 to May/2006. We considered the years as a graduate and a specialist, knowledge of anatomical nomenclature and specialty reference review. It was then compared with a repeat survey of 92 specialist physicians in General Surgery during July 2010 to July 2011. Of the 96 medical specialists in General Surgery, respondents in the first period $30(31.25 \%)$ were unaware of an official list of names of anatomical structures and 66 $(68.75 \%)$ knew of its existence. Of these, 66 were aware of an official list, $60(90.91 \%)$ presented difficulties in naming the listing and 6 $(9.09 \%)$ correctly enunciated the IAT. Of the 92 medical specialists in General Surgery respondents in the second period, $9(9.78 \%)$ were unaware of an official list of names of anatomical structures and 83 $(90.22 \%)$ knew of its existence. By questioning the 83 on the official name of the listing of names of anatomical structures, 32 (38.55\%) had difficulty naming the listing and $51(61.45 \%)$ correctly named the AIT. In reference to General Surgery, we have seen that in time the number of medical specialists who learned of the existence of an official list of names of anatomical structures, has increased. Furthermore, they have also begun to implement the International Anatomical Terminology as the official source of anatomical terms, however, a majority still remains who are unaware of its existence. It 
is therefore, necessary to work in this area, in order to encourage and achieve permanent update, unify terms and facilitate teaching and learning. It is also important to avoid confusion in scientific communication between different parts of the world and among specialists of different age groups and groups of different graduating years. It is those who specialize in morphological disciplines as well as those who apply them in everyday activities who are responsible for disseminating the information.

KEY WORDS: International Anatomical Terminology; Medical surgeon; General surgery; Level of knowledge; Anatomical terms.

\section{REFERENCIAS BIBLIOGRÁFICAS}

Acuña, M.; Sinagra, A.; Pérez, M.; Macchia, E.; Manganiello, S. \& Conesa, H. Acerca de la Terminología Anatómica. Rev. Neurocir., 9(4):114-7, 2007.

Algieri, R. D.; Pró, E. A.; Ferrante, M. S. \& Forlizzi, V. A. Aplicación de las nomenclaturas en la cirugía de la pared abdominal. Rev. Hosp. Aeronáutico Central, 5(1):37-40, 2010a.

Algieri, R. D.; Califano, L.; Pró, E.; Ferrante, M. S.; Mérola, C. \& Sarti, L. Terminología Anatómica Internacional en la región cervical. Rev. Hosp. Aeronáutico Central, 5(2):41-6, $2010 \mathrm{~b}$.

Algieri, R. D.; Pró, E. A.; Forlizzi, V. \& Ferrante, M. S. Reseña de la Evolución Histórica de los Términos Anatómicos. Rev. Arg. Anat. Online, 2(4):106-11, 2011a.

Algieri, R. D.; Arribalzaga, E.; Pró, E.; Forlizzi, V. A.; Ferrante, M. S. \& Sarti, L. Aplicación de las Nomenclaturas en Anatomía Quirúrgica del Tórax. Rev. Hosp. Aeronáutico Central, 6(1):35-8, $2011 b$.

Álvarez, O. \& Campohermoso. O. Evolución histórica conceptual de la Terminología Anatómica. Rev. Cuad., 52(1):113-7, 2007.

Cruz Gutiérrez, R., Rodríguez Torres, A.; Prates, J. C.; Losardo, R. J. \& Valverde Barbato, N. Simposios Ibero Latinoamericanos de Terminología. Anatomía, Histología y Embriología. Int. J. Morphol., 28(1):333-6. 2010.

Díaz, R. J. La terminología médica: Persidad, norma y uso. Panace, 2(4):40-8, 2001.

Federative Committee on Anatomical Terminology (FCAT). Terminología Anatómica: International Anatomical Terminology. Stuttgart, Thieme, 1998. p.292.

International Anatomical Nomenclature Committee. Nómina Anatómica $2^{a}$ ed. Aprobada en el $6^{\circ}$ y $7^{\circ}$ International Congresses of Anatomists, París (1955) y New York (1960). Amsterdam, Excerpta Medica Foundation, 1961.
International Anatomical Nomenclature Committee. Nómina Anatómica. $3^{a}$ ed. Aprobada en el $6^{\circ}$ International Congress of Anatomists, París (1955) y revisiones en el $7^{\circ}$ y $8^{\circ}$ International Congress of Anatomists, New York (1960) y Wiesbaden (1965). Amsterdam, Excerpta Medica Foundation, 1968.

International Anatomical Nomenclature Committee. Nómina Anatómica. $6^{\mathrm{a}}$ ed. Londres, $12^{\circ}$ International Congress of Anatomists, 1989.

Jiménez, G. I. La sinonimia y la polisemia en la terminología anatómica: términos de ubicación y de relación de estructuras anatómicas. Entreculturas, 1:579-97, 2009.

Manzanares-Céspedes, M. C. On-line Terminologia Anatomica: A Proposal. Anat. Sci. Educ., 3(3):154-5, 2010.

Martin, B. D.; Thorpe, D.; Barnes, R.; DeLeón, M. \& Hill, D. Frequency in usage of FCAT-Approved Anatomical Term by North American Anatomists. Anat. Sci. Educ., 2(3):94-106, 2009.

Martin, B. D.; Thorpe, D.; Merenda, V.; Finch, B.; Anderson-Smith, W. \& Consiglio-Lahti, Z. Contrast in Usage of FCAT-Approved Anatomical Terminology Between Members of Two Anatomy Associations in North America. Anat. Sci. Educ., 3(1):25-32, 2010.

Pawlina, W. \& Drake, R. Moving forward with Terminologia Anatomica. Anat. Sci. Educ., 2(3):93, 2009.

Matusz, P. Misleading Latin/English Equivalents for Some Liver Terms In Terminología Anatomica. Anat. Sci. Educ., 3(3):1567, 2010 .

Salgado, A. G. E. \& Trujillo, H. E. Terminología: Historia e importancia en el desarrollo de las ciencias morfológicas. FOPJ, 1(1):9-11, 2010.

Vogl, A. W. Awareness of and Access to a Inified Terminology by Anatomists. Anat. Sci. Educ., 2(3):139-40, 2009.

Whitemore, I. Terminologia Anatomica includes terms in English for all scientists writing in English. Anat. Sci. Educ., 2(3):141, 2009.

W-ster, E. Introducción a la teoría de la terminología y a la lexicografía terminológica. Barcelona, IULA, 1998.

Dirección para Correspondencia:

Rubén Daniel Algieri

III Cátedra de Anatomía

Facultad de Medicina

Universidad de Buenos Aires

Buenos Aires

Recibido : 02-11-2013

ARGENTINA

Aceptado: 16-03-2013

Email: rdalgieri08@hotmail.com 